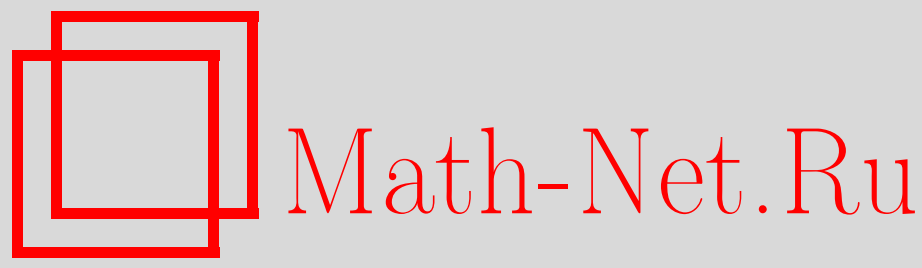

Г. В. Парадеженко, Н. Б. Мельников, Б. И. Резер, Фактор Дебая-Валлера в рассеянии нейтронов ферромагнитными металлами, ТМФ, 2018, том 195, номер 1, 91104

DOI: https://doi.org/10.4213/tmf9374

Использование Общероссийского математического портала Math-Net.Ru подразумевает, что вы прочитали и согласны с пользовательским соглашением http://www.mathnet.ru/rus/agreement

Параметры загрузки:

IP : 54.205 .225 .156

26 апреля 2023 г., 06:51:55

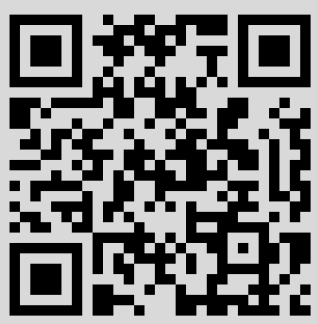




\title{
ФАКТОР ДЕБАЯ-ВАЛЛЕРА В РАССЕЯНИИ НЕЙТРОНОВ ФЕРРОМАГНИТНЫМИ МЕТАЛЛАМИ
}

\begin{abstract}
Получено выражение для сечения рассеяния нейтронов в случае произвольного взаимодействия нейтрона с кристаллом. Дан краткий и простой вывод формулы для фактора Дебая-Валлера как функции вектора рассеяния и температуры. Для ферромагнитных металлов выше температуры Кюри получена оценка фактора Дебая-Валлера в диапазоне значений вектора рассеяния, характерных для эксперимента по поляризованному магнитному рассеянию нейтронов. На примере железа проведено сравнение результатов гармонического и ангармонического приближений.
\end{abstract}

Ключевые слова: рассеяние нейтронов, колебания решетки, фактор Дебая-Валлера, корреляция спиновой плотности, ферромагнитные металлы.

DOI: https://doi.org/10.4213/tmf9374

\section{1. ВВЕДЕНИЕ}

Эксперимент по рассеянию нейтронов используется для исследования ближнего магнитного порядка в металлах, который описывается коррелятором спиновой плотности (см., например, монографии [1]-[3]). Однако в эксперименте измеряется не сам коррелятор, а его фурье-образ. Непосредственное восстановление коррелятора лишь на основе данных эксперимента неустойчиво по отношению к погрешностям измерений (подробнее см. работу [4]). Интерпретация эксперимента по поляризованному магнитному рассеянию нейтронов в рамках теории спиновых волн дает большу́ю (примерно 15-20 А) область ближнего порядка в ферромагнитных металлах в парамагнитной области [5], но является спорной (см., например, [6], [7]).

Работа выполнена в рамках государственного задания ФАНО России (тема “Электрон” № 01201463326) при частичной поддержке УрО РАН (проект № 15-8-2-10).

* Московский государственный университет им. М. В. Ломоносова, Москва, Россия. E-mail: gparadezhenko@cs.msu.su,melnikov@cs.msu.su

${ }^{\dagger}$ Институт физики металлов им. М. Н. Михеева УрО РАН, Екатеринбург, Россия. E-mail: reser@imp.uran.ru 
В рамках динамической теории спиновых флуктуаций (ДТСФ) [8] коррелятор спиновой плотности и его фурье-образ удается вычислить явно при различных температурах [9]-[11]. В наших работах [4], [10], [11] показано, что область ближнего порядка сравнительно мала (составляет несколько А), а расчеты фурье-образа коррелятора хорошо согласуются с экспериментом по поляризованному рассеянию нейтронов в парамагнитной области [5].

Связь между сечением магнитного рассеяния нейтронов и коррелятором спиновой плотности была установлена в работе [12]. Выражение для сечения неполяризованного магнитного рассеяния для магнетиков с коллективизированными электронами было выведено в нашей работе [11]. Сечение рассеяния для произвольного взаимодействия нейтрона с кристаллом рассмотрено в книге [13], но среднее по спиновым состояниям нейтрона там вычислено только для потенциала взаимодействия спина нейтрона и спинов электронов. В настоящей работе использовано представление для потенциала общего вида, существенно упрощающее вычисление среднего по спиновым состояниям. Рассмотренный нами потенциал может описывать ядерное взаимодействие, а также взаимодействия между спином нейтрона и спином атома, орбитальным током и спином электрона.

Учет колебаний решетки приводит к поправочному множителю в формуле для сечения рассеяния. Множитель, отвечающий упругому рассеянию на фононах, называется фактором Дебая-Валлера (ФДВ). В гармоническом приближении выражение для ФДВ было получено отдельно для ядерного (см., например, книги [13], [14]) и магнитного [11] типов рассеяния. В настоящей работе предложен краткий и простой способ вычисления ФДВ в случае рассеяния произвольного типа. Каноническое среднее для экспонент от операторов, линейных по смещениям, вычисляется по формуле, полученной в работе [15]. Предложенный способ позволяет опустить громоздкое вычисление среднего квадрата смещения на узле, которое возникало в выражении для ФДВ в работах [14], [11]. Подобное вычисление необходимо лишь при учете одно- и многофононного вкладов, которые, как правило, не дают полезной информации [14].

ФДВ зависит от температуры и модуля вектора рассеяния. В экспериментах по исследованию магнитных свойств металлов характерный диапазон значений вектора рассеяния составляет от $0.1 \AA^{-1}$ до $10 \AA^{-1}$ [16]. В этом диапазоне при $T=0$ ФДВ в металлах дает поправку в пределах $10 \%$ [17], и она заметно растет при конечных температурах. Так, в брэгговском рассеянии нейтронов для меди поправка за счет ФДВ при $T=10 \mathrm{~K}$ составляет около $2 \%$, а при $T=1000 \mathrm{~K}$ - около $18 \%$ (см. книгу [14]). Однако теоретические оценки, как правило, относятся к конкретным значениям вектора рассеяния, а экспериментальные измерения ФДВ в литературе отсутствуют.

В настоящей работе ФДВ для ферромагнитных металлов вычислен как функция модуля вектора рассеяния и температуры. Исследована величина поправки за счет ФДВ в эксперименте по поляризованному рассеянию нейтронов ферромагнитными металлами выше температуры Кюри [5], [18], [19]. Полученные результаты имеют универсальный характер и могут быть использованы для анализа экспериментов по рассеянию нейтронов ферромагнитными металлами.

Вклад ангармонических колебаний в ФДВ был рассмотрен в работе [20], где на примере свинца было показано, что для комнатных температур он незначите- 
лен. В настоящей работе проведено сравнение ФДВ в гармоническом приближении с результатами для железа [21], [22], полученными в рамках ангармонической теории [23]. Показано, что при температурах выше температуры Кюри и при больши́х значениях вектора рассеяния ангармонический вклад может быть заметным. Однако метод, предложенный в работе [20], требует громоздких вычислений, а его упрощения в значительной мере зависят от используемых приближений и параметров (см., например, статьи [23], [24]).

Изложение построено следующим образом. В разделе 2 получено выражение для ФДВ в нейтронном рассеянии на произвольном потенциале. В разделе 3 дана оценка ФДВ для ферромагнитных металлов в эксперименте по поляризованному магнитному рассеянию нейтронов в парамагнитной области. В разделе 4 суммированы полученные результаты.

\section{2. ФДВ В РАССЕЯНИИ НЕЙТРОНОВ НА ПРОИЗВОЛЬНОМ ПОТЕНЦИАЛЕ ВЗАИМОДЕЙСТВИЯ}

2.1. Дифференциальное сечение в борновском приближении. Количественной характеристикой процесса рассеяния является дважды дифференциальное сечение рассеяния (см., например, [14])

$$
\frac{d^{2} \sigma}{d \Omega^{\prime} d E_{k^{\prime}}}=\frac{1}{\Phi d \Omega^{\prime} d E_{k^{\prime}}}\left(\begin{array}{c}
\text { число нейтронов, рассеянных в единицу времени } \\
\text { в телесный угол } d \Omega^{\prime}, \text { с энергией } \\
\text { в конечном состоянии между } E_{k^{\prime}} \text { и } E_{k^{\prime}}+d E_{k^{\prime}}
\end{array}\right),
$$

где $\Phi$ - плотность потока падающих нейтронов, которая определяется как число нейтронов, пролетающих через единицу площади в единицу времени (здесь и далее штрихом мы обозначаем величины после рассеяния). Число нейтронов в числителе выражения (1) равно $w_{\mathbf{k} \rightarrow \mathbf{k}^{\prime}} d N\left(E_{k^{\prime}}\right)$, где $w_{\mathbf{k} \rightarrow \mathbf{k}^{\prime}}=d P_{\mathbf{k} \rightarrow \mathbf{k}^{\prime}}(t) / d t$ (здесь $P_{\mathbf{k} \rightarrow \mathbf{k}^{\prime}}(t)-$ вероятность перехода нейтрона из состояния $\mathbf{k}$ в состояние $\left.\mathbf{k}^{\prime}\right)$, а $N\left(E_{k^{\prime}}\right)$ - число состояний нейтрона с волновым вектором $\mathbf{k}^{\prime}$, лежащим в телесном угле $d \Omega^{\prime}$, с энергией, не превосходящей $E_{k^{\prime}}$. В результате дифференциальное сечение рассеяния (1) принимает вид

$$
\frac{d^{2} \sigma}{d \Omega^{\prime} d E_{k^{\prime}}}=\frac{n\left(E_{k^{\prime}}\right)}{\Phi d \Omega^{\prime}} w_{\mathbf{k} \rightarrow \mathbf{k}^{\prime}},
$$

где $n\left(E_{k^{\prime}}\right)=d N\left(E_{k^{\prime}}\right) / d E_{k^{\prime}}$ - плотность нейтронных состояний. Используя определение величины $w_{\mathbf{k} \rightarrow \mathbf{k}^{\prime}}$ и формулу полной вероятности, имеем

$$
w_{\mathbf{k} \rightarrow \mathbf{k}^{\prime}}=\sum_{\sigma, \lambda} P_{\sigma \lambda} \sum_{\sigma^{\prime}, \lambda^{\prime}} w_{\mathbf{k} \sigma \lambda \rightarrow \mathbf{k}^{\prime} \sigma^{\prime} \lambda^{\prime}}
$$

где $\sigma$ - спиновое состояние нейтрона $(\sigma=\uparrow, \downarrow), \lambda$ - состояние кристалла, а $P_{\sigma \lambda}$ - вероятность начального состояния $\sigma, \lambda$. В первом приближении нестационарной теории возмущений величина $w_{\mathbf{k} \sigma \lambda \rightarrow \mathbf{k}^{\prime} \sigma^{\prime} \lambda^{\prime}}$ задается формулой

$$
w_{\mathbf{k} \sigma \lambda \rightarrow \mathbf{k}^{\prime} \sigma^{\prime} \lambda^{\prime}}=\frac{2 \pi}{\hbar}\left|\left\langle\mathbf{k}^{\prime} \sigma^{\prime} \lambda^{\prime}|V(\mathbf{r})| \mathbf{k} \sigma \lambda\right\rangle\right|^{2} \delta\left(E_{\lambda}-E_{\lambda^{\prime}}+E_{k}-E_{k^{\prime}}\right) .
$$

Здесь $V(\mathbf{r})$ - потенциал взаимодействия нейтрона с кристаллом, $E_{\lambda}-$ энергия кристалла и $E_{k}-$ энергия нейтрона. 
В борновском приближении состояния нейтрона $\mathbf{k}$ описываются плоскими волнами $\psi_{\mathbf{k}}(\mathbf{r})=\mathcal{V}^{-1 / 2} \mathrm{e}^{i \mathbf{k r}}$, где $\mathcal{V}$ - объем кристалла, и матричный элемент принимает вид

$$
\left\langle\mathbf{k}^{\prime} \sigma^{\prime} \lambda^{\prime}|V(\mathbf{r})| \mathbf{k} \sigma \lambda\right\rangle=\frac{1}{\mathcal{V}}\left\langle\sigma^{\prime} \lambda^{\prime}\left|V_{-\kappa}\right| \sigma \lambda\right\rangle,
$$

где $V_{\boldsymbol{\kappa}}=\int V(\mathbf{r}) \mathrm{e}^{-i \boldsymbol{\kappa} \mathbf{r}} d \mathbf{r}-$ фурье-образ потенциала, а $\boldsymbol{\kappa}=\mathbf{k}-\mathbf{k}^{\prime}$ - вектор рассеяния. С учетом равенства $E_{k}=\hbar^{2} k^{2} / 2 m$ плотность нейтронных состояний задается выражением

$$
n\left(E_{k^{\prime}}\right)=\frac{\mathcal{V} k^{\prime} m d \Omega^{\prime}}{\hbar^{2}(2 \pi)^{3}}
$$

где $m$ - масса нейтрона. Плотность потока падающих нейтронов вычисляется по формуле

$$
\mathbf{\Phi}=\frac{i \hbar}{2 m}\left(\psi_{\mathbf{k}} \nabla \psi_{\mathbf{k}}^{*}-\psi_{\mathbf{k}}^{*} \nabla \psi_{\mathbf{k}}\right)=\frac{\hbar \mathbf{k}}{\mathcal{V} m} .
$$

В результате, используя (3)-(7), получаем дифференциальное сечение (2) в борновском приближении:

$$
\frac{d^{2} \sigma}{d \Omega^{\prime} d E_{k^{\prime}}}=\frac{k^{\prime}}{k}\left(\frac{m}{2 \pi \hbar^{2}}\right)^{2} \sum_{\sigma, \lambda} P_{\sigma \lambda} \sum_{\sigma^{\prime}, \lambda^{\prime}}\left|\left\langle\sigma^{\prime} \lambda^{\prime}\left|V_{-\kappa}\right| \sigma \lambda\right\rangle\right|^{2} \delta\left(E_{\lambda}-E_{\lambda^{\prime}}+E_{k}-E_{k^{\prime}}\right) .
$$

2.2. Коррелятор потенциала взаимодействия. Предположим, что потенциал $V(\mathbf{r})$ можно представить как

$$
V(\mathbf{r})=W(\mathbf{r}) U
$$

где $W(\mathbf{r})$ - оператор, действующий на состояния нейтрона $\mathbf{k}$ и состояния кристалла $\lambda$, а $U$ - оператор, действующий на спиновые состояния нейтрона $\sigma$. Используя представление эрмитовой $(2 \times 2)$-матрицы $U$ в виде

$$
U=\sum_{\mu} U^{\mu} \sigma^{\mu}, \quad U^{\mu}=\frac{1}{2} \operatorname{Tr}\left(U \sigma^{\mu}\right), \quad \mu=0, x, y, z,
$$

где $\sigma^{0}$ - единичная $(2 \times 2)$-матрица и $\sigma^{x}, \sigma^{y}, \sigma^{z}$ - матрицы Паули, записываем потенциал (9) как

$$
V(\mathbf{r})=\sum_{\mu} V^{\mu}(\mathbf{r}) \sigma^{\mu}, \quad V^{\mu}(\mathbf{r})=U^{\mu} W(\mathbf{r})
$$

Потенциал такого вида описывает произвольное взаимодействие нейтрона с кристаллом, включая ядерное взаимодействие, взаимодействия между спином нейтрона и спином атома, орбитальным током и спином электрона.

Независимость состояний $\sigma$ и $\lambda$ приводит к следующим упрощениям. Во-первых, матричный элемент потенциала (10) распадается на независимые множители:

$$
\left\langle\sigma^{\prime} \lambda^{\prime}\left|V_{-\kappa}\right| \sigma \lambda\right\rangle=\sum_{\mu}\left\langle\sigma^{\prime} \lambda^{\prime}\left|V_{-\kappa}^{\mu} \sigma^{\mu}\right| \sigma \lambda\right\rangle=\sum_{\mu}\left\langle\lambda^{\prime}\left|V_{-\kappa}^{\mu}\right| \lambda\right\rangle\left\langle\sigma^{\prime}\left|\sigma^{\mu}\right| \sigma\right\rangle
$$


Во-вторых, для вероятности справедливо равенство $P_{\sigma \lambda}=P_{\sigma} P_{\lambda}$. В результате формула (8) принимает вид

$$
\begin{gathered}
\frac{d^{2} \sigma}{d \Omega^{\prime} d E_{k^{\prime}}}=\frac{k^{\prime}}{k}\left(\frac{m}{2 \pi \hbar^{2}}\right)^{2} \sum_{\mu, \nu} \sum_{\lambda} P_{\lambda} \sum_{\lambda^{\prime}}\left\langle\lambda\left|V_{\kappa}^{\mu}\right| \lambda^{\prime}\right\rangle\left\langle\lambda^{\prime}\left|V_{-\kappa}^{\nu}\right| \lambda\right\rangle \delta\left(E_{\lambda}-E_{\lambda^{\prime}}+E_{k}-E_{k^{\prime}}\right) \times \\
\times \sum_{\sigma} P_{\sigma} \sum_{\sigma^{\prime}}\left\langle\sigma\left|\sigma^{\mu}\right| \sigma^{\prime}\right\rangle\left\langle\sigma^{\prime}\left|\sigma^{\nu}\right| \sigma\right\rangle .
\end{gathered}
$$

Сначала вычислим среднее по состояниям со спином $\sigma$ и сумму по состояниям со спином $\sigma^{\prime}$ в выражении (11). Используя формулу для матричного элемента произведения матриц, получаем

$$
\sum_{\sigma} P_{\sigma} \sum_{\sigma^{\prime}}\left\langle\sigma\left|\sigma^{\mu}\right| \sigma^{\prime}\right\rangle\left\langle\sigma^{\prime}\left|\sigma^{\nu}\right| \sigma\right\rangle=\sum_{\sigma} P_{\sigma}\left\langle\sigma\left|\sigma^{\mu} \sigma^{\nu}\right| \sigma\right\rangle
$$

Для пучка неполяризованных нейтронов $\left(P_{\uparrow}=P_{\downarrow}=1 / 2\right)$ справедливо равенство

$$
\sum_{\sigma} P_{\sigma}\left\langle\sigma\left|\sigma^{\mu} \sigma^{\nu}\right| \sigma\right\rangle=\delta_{\mu \nu}, \quad \mu, \nu=0, x, y, z
$$

Подставляя (12) в формулу (11), с учетом последнего соотношения имеем

$$
\frac{d^{2} \sigma}{d \Omega^{\prime} d E_{k^{\prime}}}=\frac{k^{\prime}}{k}\left(\frac{m}{2 \pi \hbar^{2}}\right)^{2} \sum_{\mu} \sum_{\lambda} P_{\lambda} \sum_{\lambda^{\prime}}\left\langle\lambda\left|V_{\kappa}^{\mu}\right| \lambda^{\prime}\right\rangle\left\langle\lambda^{\prime}\left|V_{-\kappa}^{\mu}\right| \lambda\right\rangle \delta\left(E_{\lambda}-E_{\lambda^{\prime}}+E_{k}-E_{k^{\prime}}\right) .
$$

Теперь вычисляем среднее по состояниям $\lambda$ и сумму по состояниям $\lambda^{\prime}$ в формуле (13). Используя соотношение

$$
\delta\left(E_{\lambda}-E_{\lambda^{\prime}}+\hbar \omega\right)=\frac{1}{2 \pi \hbar} \int \mathrm{e}^{i\left(E_{\lambda}-E_{\lambda^{\prime}}\right) t / \hbar} \mathrm{e}^{i \omega t} d t
$$

где $\hbar \omega=E_{k}-E_{k^{\prime}}$, записываем выражение (13) в виде

$$
\frac{d^{2} \sigma}{d \Omega^{\prime} d E_{k^{\prime}}}=\frac{k^{\prime}}{k}\left(\frac{m}{2 \pi \hbar^{2}}\right)^{2} \sum_{\mu} \frac{1}{2 \pi \hbar} \int \sum_{\lambda} P_{\lambda} \sum_{\lambda^{\prime}}\left\langle\lambda\left|V_{\kappa}^{\mu} \mathrm{e}^{-i E_{\lambda^{\prime}} t / \hbar}\right| \lambda^{\prime}\right\rangle\left\langle\lambda^{\prime}\left|V_{-\kappa}^{\mu} \mathrm{e}^{i E_{\lambda} t / \hbar}\right| \lambda\right\rangle \mathrm{e}^{i \omega t} d t .
$$

Выбирая в качестве состояний $\lambda$ собственные функции гамильтониана кристалла,

$$
H|\lambda\rangle=E_{\lambda}|\lambda\rangle, \quad \mathrm{e}^{-i H t / \hbar}|\lambda\rangle=\mathrm{e}^{-i E_{\lambda} t / \hbar}|\lambda\rangle,
$$

приходим к выражению

$$
\frac{d^{2} \sigma}{d \Omega^{\prime} d E_{k^{\prime}}}=\frac{k^{\prime}}{k}\left(\frac{m}{2 \pi \hbar^{2}}\right)^{2} \sum_{\mu} \frac{1}{2 \pi \hbar} \int \sum_{\lambda} P_{\lambda}\left\langle\lambda\left|\mathrm{e}^{i H t / \hbar} V_{\kappa}^{\mu} \mathrm{e}^{-i H t / \hbar} V_{-\kappa}^{\mu}\right| \lambda\right\rangle \mathrm{e}^{i \omega t} d t .
$$

Сумма по $\lambda$ есть не что иное, как каноническое среднее

$$
\langle\cdot\rangle \equiv \frac{1}{Z} \operatorname{Tr}\left(\cdot \mathrm{e}^{-H / T}\right)=\sum_{\lambda} P_{\lambda}\langle\lambda|\cdot| \lambda\rangle
$$


где $Z=\operatorname{Tr} \mathrm{e}^{-H / T}$ - статистическая сумма, $P_{\lambda}=Z^{-1} \mathrm{e}^{-E_{\lambda} / T}$ и $T$ - температура (в энергетических единицах). Используя представление Гейзенберга $V_{\kappa}^{\mu}(t)=$ $\mathrm{e}^{i H t / \hbar} V_{\kappa}^{\mu} \mathrm{e}^{-i H t / \hbar}$, получаем окончательную формулу

$$
\frac{d^{2} \sigma}{d \Omega^{\prime} d E_{k^{\prime}}}=\frac{k^{\prime}}{k}\left(\frac{m}{2 \pi \hbar^{2}}\right)^{2} \sum_{\mu} \frac{1}{2 \pi \hbar} \int\left\langle V_{\kappa}^{\mu}(t) V_{-\kappa}^{\mu}\right\rangle \mathrm{e}^{i \omega t} d t
$$

связывающую дифференциальное сечение с коррелятором потенциала.

2.3. Учет колебаний решетки в гармоническом приближении. Предположим теперь, что каждый узел может смещаться на вектор $\mathbf{u}_{j}$ из положения равновесия $\mathbf{R}_{j}$. Заменяя $\mathbf{R}_{j}$ на $\mathbf{R}_{j}+\mathbf{u}_{j}$, записываем фурье-образ потенциала в виде

$$
V_{\boldsymbol{\kappa}}^{\mu}=\sum_{j} V_{j}^{\mu} \mathrm{e}^{-i \boldsymbol{\kappa}\left(\mathbf{R}_{j}+\mathbf{u}_{j}\right)}, \quad V_{j}^{\mu}=\int_{\Omega_{j}} V^{\mu}(\mathbf{r}) d \mathbf{r},
$$

где $\Omega_{j}$ - ячейка Вигнера-Зейтца с центром $\mathbf{R}_{j}$. С учетом (15) коррелятор потенциала принимает вид

$$
\left\langle V_{\kappa}^{\mu}(t) V_{-\boldsymbol{\kappa}}^{\mu}\right\rangle=\sum_{j, j^{\prime}} \mathrm{e}^{-i \boldsymbol{\kappa}\left(\mathbf{R}_{j}-\mathbf{R}_{j^{\prime}}\right)}\left\langle V_{j}^{\mu}(t) \mathrm{e}^{-i \kappa \mathbf{u}_{j}(t)} V_{j^{\prime}}^{\mu} \mathrm{e}^{i \kappa \mathbf{u}_{j^{\prime}}}\right\rangle .
$$

Предполагая, что колебания узлов и взаимодействие нейтрона с кристаллом происходят независимо, разбиваем среднее на произведение средних:

$$
\left\langle V_{j}^{\mu}(t) \mathrm{e}^{-i \kappa \mathbf{u}_{j}(t)} V_{j^{\prime}}^{\mu} \mathrm{e}^{i \kappa \mathbf{u}_{j^{\prime}}}\right\rangle=\left\langle V_{j}^{\mu}(t) V_{j^{\prime}}^{\mu}\right\rangle\left\langle\mathrm{e}^{-i \kappa \mathbf{u}_{j}(t)} \mathrm{e}^{i \kappa \mathbf{u}_{j^{\prime}}}\right\rangle .
$$

Введем для краткости обозначения $A=-i \boldsymbol{\kappa} \mathbf{u}_{j}(t)$ и $B=i \boldsymbol{\kappa} \mathbf{u}_{j^{\prime}}$ и подставим (16) в формулу (14). Тогда с учетом (17) имеем

$$
\frac{d^{2} \sigma}{d \Omega^{\prime} d E_{k^{\prime}}}=\frac{k^{\prime}}{k}\left(\frac{m}{2 \pi \hbar^{2}}\right)^{2} \sum_{j, j^{\prime}} \mathrm{e}^{-i \boldsymbol{\kappa}\left(\mathbf{R}_{j}-\mathbf{R}_{j^{\prime}}\right)} \sum_{\mu} \frac{1}{2 \pi \hbar} \int\left\langle V_{j}^{\mu}(t) V_{j^{\prime}}^{\mu}\right\rangle\left\langle\mathrm{e}^{A} \mathrm{e}^{B}\right\rangle \mathrm{e}^{i \omega t} d t .
$$

Преобразуем выражение $\left\langle\mathrm{e}^{A} \mathrm{e}^{B}\right\rangle$. Оператор смещения на узле $\mathbf{u}_{j}(t)$ задается выражением (см., например, монографию [14])

$$
\mathbf{u}_{j}(t)=\left(\frac{\hbar}{2 M N}\right)^{1 / 2} \sum_{s} \frac{\mathbf{e}_{s}}{\sqrt{\omega_{s}}}\left(b_{s} \mathrm{e}^{i\left(\mathbf{q} \mathbf{R}_{j}-\omega_{s} t\right)}+b_{s}^{\dagger} \mathrm{e}^{-i\left(\mathbf{q} \mathbf{R}_{j}-\omega_{s} t\right)}\right),
$$

где $M$ - масса атома, $N$ - число узлов кристаллической решетки, $b_{s}^{\dagger}$ и $b_{s}-$ операторы рождения и уничтожения, $\omega_{s}$ - частота колебаний и $\mathbf{e}_{s}-$ вектор поляризации $s$-й нормальной моды. С учетом (19) операторы $A$ и $B$ принимают вид

$$
A=-i \sum_{s}\left(\alpha_{s} b_{s}+\alpha_{s}^{*} b_{s}^{\dagger}\right), \quad B=i \sum_{s}\left(\beta_{s} b_{s}+\beta_{s}^{*} b_{s}^{\dagger}\right)
$$

где

$$
\alpha_{s}=\left(\frac{\hbar}{2 M N}\right)^{1 / 2} \frac{\boldsymbol{\kappa} \mathbf{e}_{s}}{\sqrt{\omega_{s}}} \mathrm{e}^{i\left(\mathbf{q} \mathbf{R}_{j}-\omega_{s} t\right)}, \quad \beta_{s}=\left(\frac{\hbar}{2 M N}\right)^{1 / 2} \frac{\boldsymbol{\kappa} \mathbf{e}_{s}}{\sqrt{\omega_{s}}} \mathrm{e}^{i \mathbf{q} \mathbf{R}_{j^{\prime}}}
$$


Поскольку коммутатор $[A, B]=\sum_{s}\left(\alpha_{s} \beta_{s}^{*}-\alpha_{s}^{*} \beta_{s}\right)$ пропорционален единичному оператору, справедлива формула (см., например, монографию [14])

$$
\mathrm{e}^{A} \mathrm{e}^{B}=\mathrm{e}^{A+B} \mathrm{e}^{[A, B] / 2}
$$

Смещения $\mathbf{u}_{j}$ имеют гауссово распределение ${ }^{1)}$, следовательно, гауссово распределение имеют также величины $A$ и $B$, и для них справедливы соотношения $\left\langle\mathrm{e}^{A}\right\rangle=$ $\mathrm{e}^{\left\langle A^{2}\right\rangle / 2},\left\langle\mathrm{e}^{B}\right\rangle=\mathrm{e}^{\left\langle B^{2}\right\rangle / 2}$ и $\left\langle\mathrm{e}^{A+B}\right\rangle=\mathrm{e}^{\left\langle(A+B)^{2}\right\rangle / 2}$ (см., например, книгу [26]). Тогда каноническое среднее выражения (22) принимает вид

$$
\left\langle\mathrm{e}^{A} \mathrm{e}^{B}\right\rangle=\mathrm{e}^{\left\langle(A+B)^{2}\right\rangle / 2} \mathrm{e}^{\langle[A, B]\rangle / 2}=\mathrm{e}^{\left\langle A^{2}\right\rangle / 2} \mathrm{e}^{\left\langle B^{2}\right\rangle / 2} \mathrm{e}^{\langle A B\rangle}=\left\langle\mathrm{e}^{A}\right\rangle\left\langle\mathrm{e}^{B}\right\rangle \mathrm{e}^{\langle A B\rangle} .
$$

Рассматривая вклад лишь упругого рассеяния на фононах, $\mathrm{e}^{\langle A B\rangle}=1$ (подробнее см. [14]), приходим к соотношению

$$
\left\langle\mathrm{e}^{A} \mathrm{e}^{B}\right\rangle=\left\langle\mathrm{e}^{A}\right\rangle\left\langle\mathrm{e}^{B}\right\rangle
$$

Для канонического среднего $\left\langle\mathrm{e}^{Q}\right\rangle$ с произвольным оператором $Q=\sum_{s}\left(c_{s} b_{s}+d_{s} b_{s}^{\dagger}\right)$, линейно зависящим от смещений, в гармоническом приближении справедлива формула [15]

$$
\left\langle\mathrm{e}^{Q}\right\rangle=\exp \left[\frac{1}{2} \sum_{s} c_{s} d_{s} \operatorname{cth} \frac{\hbar \omega_{s}}{2 T}\right] .
$$

Вычисляя с помощью этой формулы средние $\left\langle\mathrm{e}^{A}\right\rangle$ и $\left\langle\mathrm{e}^{B}\right\rangle$ в выражении (24), с учетом равенств (20) имеем

$$
\left\langle\mathrm{e}^{A} \mathrm{e}^{B}\right\rangle=\exp \left[-\frac{1}{2} \sum_{s}\left(\left|\alpha_{s}\right|^{2}+\left|\beta_{s}\right|^{2}\right) \operatorname{cth} \frac{\hbar \omega_{s}}{2 T}\right] .
$$

Используя (21), приходим к выражению

$$
\left\langle\mathrm{e}^{A} \mathrm{e}^{B}\right\rangle=\mathrm{e}^{-2 W(\kappa)}
$$

где

$$
2 W(\boldsymbol{\kappa})=\frac{\hbar}{2 M N} \sum_{s} \frac{\left(\boldsymbol{\kappa} \mathbf{e}_{s}\right)^{2}}{\omega_{s}} \operatorname{cth} \frac{\hbar \omega_{s}}{2 T} .
$$

Наконец, подставляя (26) в (18) и возвращаясь обратно к фурье-образу потенциала (15), получаем выражение для дифференциального сечения рассеяния:

$$
\frac{d^{2} \sigma}{d \Omega^{\prime} d E_{k^{\prime}}}=\frac{k^{\prime}}{k}\left(\frac{m}{2 \pi \hbar^{2}}\right)^{2} \mathrm{e}^{-2 W(\kappa)} \sum_{\mu} \frac{1}{2 \pi \hbar} \int\left\langle V_{\kappa}^{\mu}(t) V_{-\kappa}^{\mu}\right\rangle \mathrm{e}^{i \omega t} d t .
$$

Экспонента $\mathrm{e}^{-2 W(\kappa)}$ и называется ФДВ. Как видно из (27), ФДВ зависит от вектора рассеяния и температуры.

\footnotetext{
1) Простое доказательство этого факта дано в нашей статье [25].
} 


\section{3. ФДВ В ПОЛЯРИЗОВАННОМ МАГНИТНОМ РАССЕЯНИИ}

Магнитное рассеяние в металлах определяется взаимодействием между спином нейтрона и спинами электронов ${ }^{2)}$. Фурье-образ потенциала магнитного взаимодействия имеет вид (подробнее см. [11])

$$
V_{\kappa}=\frac{2 \pi \gamma e^{2} \hbar^{2}}{m_{e} m c^{2}} \sum_{\alpha} \tilde{s}_{\kappa}^{\alpha} \sigma^{\alpha}, \quad \alpha=x, y, z,
$$

где через $m_{e}$ и $-e$ обозначены масса и заряд электрона, $c$ - скорость света, $\gamma \approx 1.913$, $\tilde{\mathbf{s}}_{\boldsymbol{\kappa}}=\mathbf{s}_{\boldsymbol{\kappa}}-\left(\hat{\boldsymbol{\kappa}} \mathbf{s}_{\boldsymbol{\kappa}}\right) \hat{\boldsymbol{\kappa}}, \mathbf{s}_{\boldsymbol{\kappa}}-$ фурье-образ оператора спиновой плотности, а $\hat{\boldsymbol{\kappa}}=\boldsymbol{\kappa} / \kappa-$ единичный вектор. Подставляя (29) в (28), получаем дифференциальное сечение неполяризованного магнитного рассеяния:

$$
\frac{d^{2} \sigma}{d \Omega^{\prime} d E_{k^{\prime}}}=N\left(\frac{\gamma e^{2}}{m_{e} c^{2}}\right)^{2} \frac{k^{\prime}}{k} \mathrm{e}^{-2 W(\kappa)} \sum_{\alpha, \beta}\left(\delta_{\alpha \beta}-\hat{\kappa}^{\alpha} \hat{\kappa}^{\beta}\right) S^{\alpha \beta}(\kappa, \omega),
$$

где

$$
S^{\alpha \beta}(\kappa, \omega)=\frac{1}{2 \pi \hbar N} \int\left\langle s_{\kappa}^{\alpha}(t) s_{-\kappa}^{\beta}\right\rangle \mathrm{e}^{i \omega t} d t, \quad \alpha, \beta=x, y, z,
$$

- функция рассеяния. В парамагнитной области мы имеем $S^{\alpha \beta}(\kappa, \omega)=S^{z}(\kappa, \omega) \delta_{\alpha \beta}$. Тогда с учетом $N=\mathcal{V} / \Omega_{\mathrm{ws}}$ (здесь $\Omega_{\mathrm{ws}}$ - объем ячейки Вигнера-Зейтца) формулу (30) можно записать в виде

$$
\frac{d^{2} \sigma}{d \Omega^{\prime} d E_{k^{\prime}}}=2\left(\frac{\gamma e^{2}}{m_{e} c^{2}}\right)^{2} \frac{1}{3} \frac{\mathcal{V}}{\Omega_{\mathrm{ws}}} \frac{k^{\prime}}{k} \mathrm{e}^{-2 W(\kappa)} S(\kappa, \omega),
$$

где $S(\kappa, \omega)=3 S^{z}(\kappa, \omega)$. Непосредственное применение формулы (31) затруднительно, поскольку в эксперименте необходимо выделить магнитный вклад в рассеяние.

В эксперименте по поляризованному магнитному рассеянию измеряется магнитный вклад в сечение рассеяния (см., например, [2]). В парамагнитной области формула для магнитного вклада принимает вид [28] (подробный вывод см. в работе [11])

$$
\left(\frac{d^{2} \sigma^{\uparrow \downarrow}}{d \Omega^{\prime} d E_{k^{\prime}}}\right)_{\|}-\left(\frac{d^{2} \sigma^{\uparrow \downarrow}}{d \Omega^{\prime} d E_{k^{\prime}}}\right)_{\perp}=\left(\frac{\gamma e^{2}}{m_{e} c^{2}}\right)^{2} \frac{1}{3} \frac{\mathcal{V}}{\Omega_{\mathrm{ws}}} \frac{k^{\prime}}{k} \mathrm{e}^{-2 W(\kappa)} S(\kappa, \omega) .
$$

В работах [5], [19], [28] описаны эксперименты, в которых измеряется суммарное сечение по всем энергиям. Интегрируя (32) по энергии $E_{k^{\prime}}=E_{k}-\hbar \omega$, получаем

$$
\left(\frac{d \sigma^{\uparrow \downarrow}}{d \Omega^{\prime}}\right)_{\|}-\left(\frac{d \sigma^{\uparrow \downarrow}}{d \Omega^{\prime}}\right)_{\perp}=\left(\frac{\gamma e^{2}}{m_{e} c^{2}}\right)^{2} \frac{1}{3} \frac{\mathcal{V}}{\Omega_{\mathrm{ws}}} \frac{k^{\prime}}{k} \mathrm{e}^{-2 W(\kappa)} M^{2}(\kappa),
$$

где

$$
M(\kappa)=\left(\hbar \int S(\kappa, \omega) d \omega\right)^{1 / 2}
$$

- эффективный магнитный момент.

${ }^{2)} \mathrm{B}$ ферромагнитных металлах спин-орбитальное взаимодействие дает малый вклад в сечение рассеяния (см., например, статью [27]). 
Вычислим ФДВ $\mathrm{e}^{-2 W(\kappa)}$ для ферромагнитных металлов. В модели Дебая при высоких температурах выражение (27) принимает вид ${ }^{3)}$ (подробнее см. статью [11])

$$
2 W(\kappa)=\frac{3 \hbar^{2} \kappa^{2}}{M} \frac{T}{\Theta_{\mathrm{D}}^{2}}, \quad T \gg \Theta_{\mathrm{D}},
$$

где $\Theta_{\mathrm{D}}$ - температура Дебая (в энергетических единицах). Значения $\Theta_{\mathrm{D}}$ определяются следующим образом. Теплоемкость решетки вычисляется как функция температуры $T$ с использованием плотности фононных состояний, полученной по экспериментальным данным, и подставляется в формулу для теплоемкости в модели Дебая (см, например, [26], [30]). Далее $\Theta_{\mathrm{D}}$ полагается неизвестным параметром и при каждом $T$ определяется "экспериментальная" температура Дебая $\Theta_{\mathrm{D}}^{\exp }(T)$. Изменение величины $\Theta_{\mathrm{D}}^{\exp }(T)$ с температурой для твердых тел достигает порядка 10\% [30]. В наших расчетах в качестве $\Theta_{\mathrm{D}}$ используется значение $\Theta_{\mathrm{D}}^{\exp }(T)$ при больших $T$, взятое из книги [31]. Исходные данные расчетов приведены в табл. 1.

ТАБлицА 1. Экспериментальная температура Кюри $T_{\mathrm{C}}^{\exp }$, температура Дебая $\Theta_{\mathrm{D}}$ и атомная масса $M$ для ферромагнитных металлов.

\begin{tabular}{l|l|l|l}
\hline Параметр & $\mathrm{Fe}$ & $\mathrm{Co}$ & $\mathrm{Ni}$ \\
\hline$T_{\mathrm{C}}^{\exp }, \mathrm{K}[32]$ & 1044 & 1390 & 631 \\
$\Theta_{\mathrm{D}}, \mathrm{K}[31]$ & 418 & 385 & 375 \\
$M$, a.e.м. [33] & 55.85 & 58.93 & 58.69 \\
\hline
\end{tabular}

ТАБлицА 2. ФДВ $\mathrm{e}^{-2 W(\kappa)}$ для ферромагнитных металлов, вычисленный с использованием формулы (33) в парамагнитной области при $\kappa=3 \AA^{-1}$.

\begin{tabular}{ll|ll|ll}
\hline$T / T_{\mathrm{C}}^{\exp }$ & $\mathrm{Fe}$ & $T / T_{\mathrm{C}}^{\exp }$ & $\mathrm{Co}$ & $T / T_{\mathrm{C}}^{\exp }$ & $\mathrm{Ni}$ \\
\hline 1.2 & 0.845 & 1.05 & 0.803 & 1.25 & 0.882 \\
1.3 & 0.833 & 1.1 & 0.795 & 1.5 & 0.860 \\
1.4 & 0.822 & 1.15 & 0.787 & 2.0 & 0.818 \\
\hline
\end{tabular}

В табл. 2 приведены рассчитанные значения ФДВ для ферромагнитных металлов выше температуры Кюри. ФДВ вычислен по формуле (33) при $\kappa=3 \AA^{-1}$, что отвечает максимальной величине вектора рассеяния в эксперименте по поляризованному магнитному рассеянию [5], [19]. Значения температур в парамагнитной области для железа и никеля соответствуют экспериментальным значениям из работ [5], [19]. Как видно из табл. 2, учет колебаний решетки за счет ФДВ вносит поправку порядка $20 \%$ для всех ферромагнитных металлов. Наши результаты находятся в согласии с поправкой для меди, равной 18\%, которая получена в работе [14] при том же значении вектора рассеяния и температуре $T=1000 \mathrm{~K}$.

\footnotetext{
3) В книге [29] расчетная формула для $W(\kappa)$ получена с помощью формулы Линдемана для температуры плавления. Этот результат позволяет оценить ФДВ по температуре плавления вместо температуры Дебая. Однако формула Линдемана позволяет получить лишь качественную оценку (см. монографию [30]).
} 


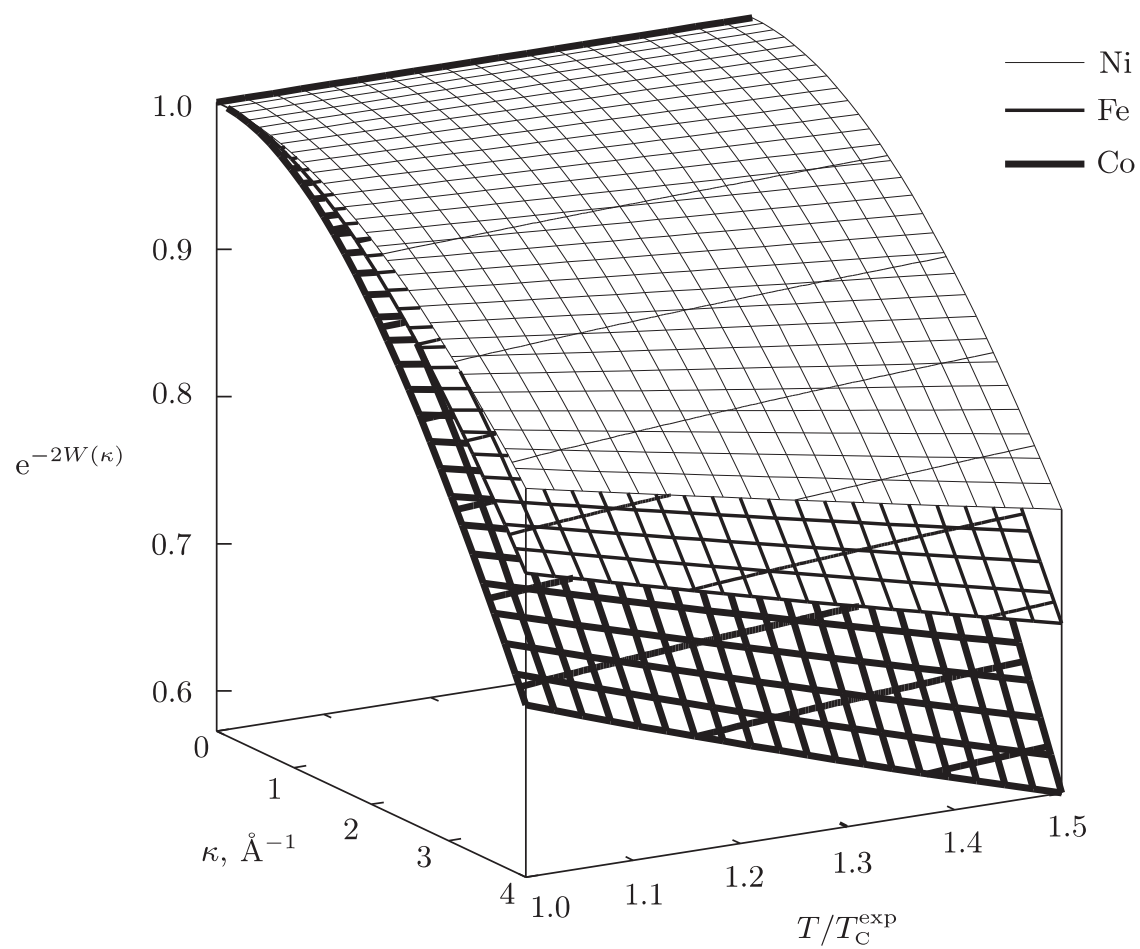

Рис. 1. ФДВ $\mathrm{e}^{-2 W(\kappa)}$ для ферромагнитных металлов, рассчитанный по формуле (33).

На рис. 1 приведены результаты расчета ФДВ по формуле (33) для ферромагнитных металлов в парамагнитной области в более широком диапазоне значений $\kappa$ от нуля до $4 \AA^{-1}$. Этот диапазон отвечает эксперименту по поляризованному рассеянию нейтронов, описанному в работе [18]. Из рис. 1 видно, что поправка составляет до $25-30 \%$ для всех ферромагнитных металлов ${ }^{4)}$. При бо́льших $\kappa$ необходим более последовательный учет фононов в металлах (см., например, монографию [34]).

Дальнейшие результаты мы иллюстрируем на примере железа. Квадрат локального магнитного момента можно вычислить по формуле

$$
m_{\mathrm{L}}^{2}=\frac{4 \pi}{\Omega_{\mathrm{BZ}}} \int_{0}^{q_{\mathrm{B}}} q^{2} M^{2}(q) d q,
$$

где $\Omega_{\mathrm{BZ}}$ - объем зоны Бриллюэна, для простоты замененной сферой равного объема

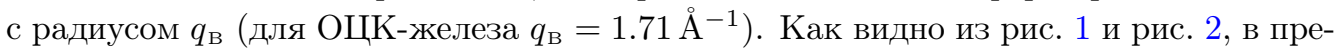
делах зоны Бриллюэна ФДВ дает несущественную поправку (для ОЦК-железа не более $4 \%$ [11]). Поэтому при вычислении локального момента на основе результатов эксперимента по рассеянию нейтронов можно опустить ФДВ (см., например, [5]). По той же причине поправка за счет колебаний решетки не играет решающей роли

\footnotetext{
${ }^{4)}$ Строго говоря, расчет величины ФДВ для кобальта справедлив лишь до температуры плавления $T / T_{\mathrm{C}}^{\exp } \approx 1.27$.
} 


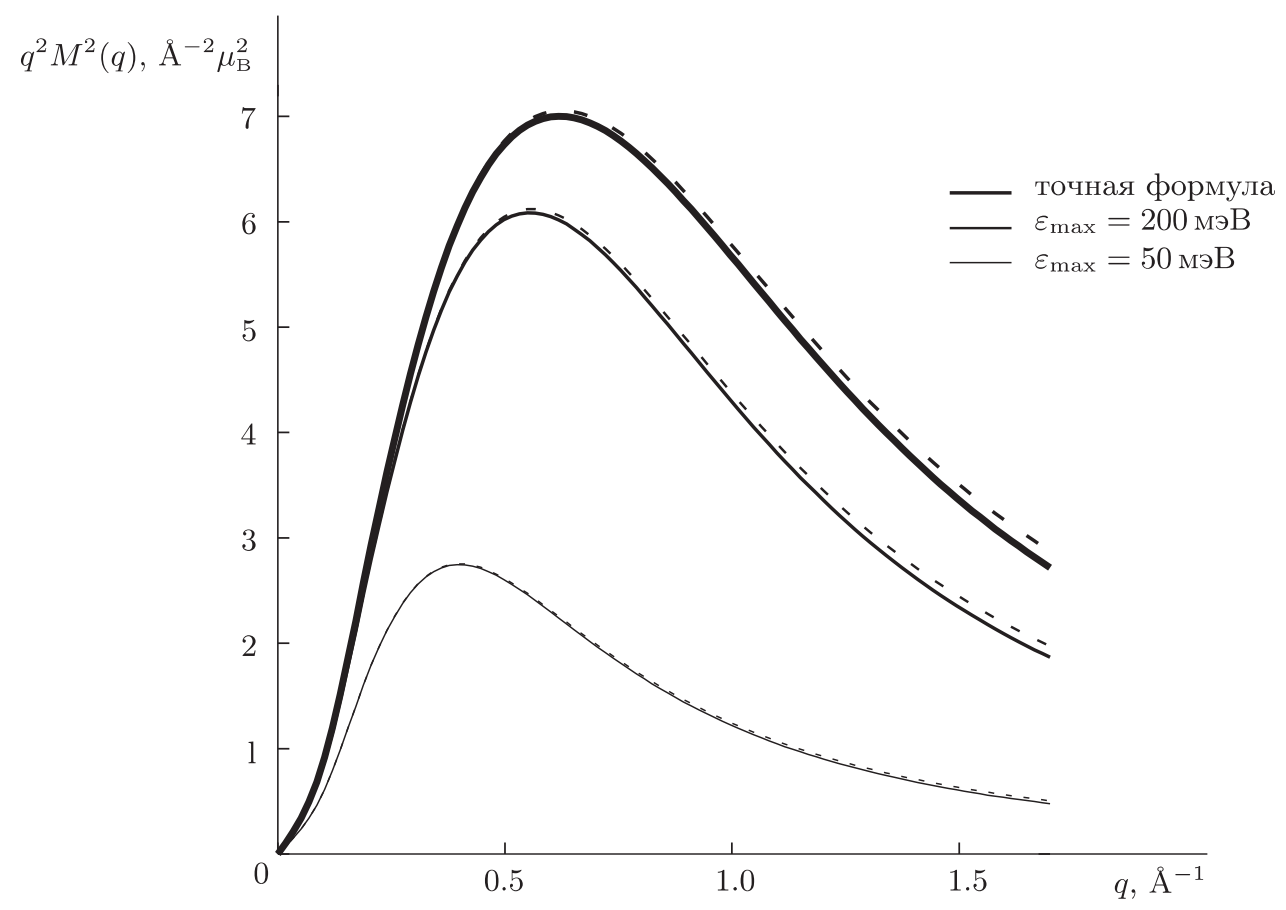

Рис. 2. Функция $q^{2} M^{2}(q)$ для ОЦК-железа при температуре $T / T_{\mathrm{C}}^{\mathrm{cal}}=1.21$, вычисленная в ДТСФ (штриховые линии) с энергетическим окном $\varepsilon_{\max }$, равным 50 и 200 мэВ, и по точной формуле. Сплошными линиями изображены результаты, умноженные на ФДВ с показателем (33).

и при расчете других магнитных характеристик, которые определяются интегралом по зоне Бриллюэна, таких как локальная восприимчивость, одноузельный спиновый коррелятор, ядерные времена релаксации и др. (см. подробности их вычисления в ДТСФ в работе [35]). Основную роль в описании магнитных свойств металлов при конечных температурах играют тепловые спиновые флуктуации.

Квадрат локального магнитного момента (34) пропорционален площади под графиком функции $q^{2} M^{2}(q)$. На рис. 2 приведены результаты расчета $q^{2} M^{2}(q)$ для ОЦК-железа в гауссовом приближении ДТСФ с энергетическим окном $\varepsilon_{\max }$, равным 50 мэВ и 200 мэВ, и по точной формуле при температуре $T / T_{\mathrm{C}}^{\mathrm{cal}}=1.21$, где $T_{\mathrm{C}}^{\mathrm{cal}}=1.65 T_{\mathrm{C}}^{\mathrm{exp}}-$ расчетная температура Кюри для ОЦК-железа (подробности вычислений см. в [11], [4]).

Локальный момент $m_{\mathrm{L}}=1.2 \mu_{\mathrm{B}}$, рассчитанный в ДТСФ с $\varepsilon_{\max }=50$ мэВ, находится в согласии с экспериментальным значением $m_{\mathrm{L}}=1.3 \mu_{\mathrm{B}}$ [5], полученным при той же температуре $T / T_{\mathrm{C}}^{\exp }$. Локальный момент, вычисленный с $\varepsilon_{\max }=200$ мэВ, получился равным $m_{\mathrm{L}}=2.0 \mu_{\mathrm{B}}$; экспериментальное значение при том же $T / T_{\mathrm{C}}^{\exp }$ равно $1.55 \mu_{\mathrm{B}}[36]$. Расчет в ДТСФ по точной формуле дает $m_{\mathrm{L}}=2.21 \mu_{\mathrm{B}}$. Этот результат находится в согласии с расчетами, использующими гамильтонианы с классическими спинами [37], [38] (подробнее см. в работе [10]). 
Оценим вклад ангармонических поправок в ФДВ для ОЦК-железа, используя результаты статей [21], [22]. В обозначениях работы [21] имеем

$$
2 W(\kappa)=\frac{\kappa^{2}}{8 \pi^{2}} B(T)
$$

где $B(T)$ - функция, пропорциональная среднеквадратичному смещению атомов. В гармоническом приближении зависимость $B$ от температуры линейная:

$$
B_{\mathrm{h}}(T)=8 \pi^{2} \alpha_{0}^{-1} T
$$

где $\alpha_{0}=8.39$ эВ $\cdot \AA^{-2}-$ параметр, определяемый из расчета ФДВ по формуле $(27)$ с реалистичной плотностью фононных состояний. В ангармоническом приближении функция $B(T)$ нелинейна и содержит эмпирический параметр, который определяется по экспериментальным данным. В работах [21], [22] приведен расчет величины

$$
Y(T)=0.86\left(B\left(T_{0}\right)-B(T)\right)
$$

где $T_{0}=286 \mathrm{~K}$, а эмпирический параметр функции $B(T)$ выбран так, чтобы кривая $Y(T)$ проходила через экспериментальное значение при $T=1321 \mathrm{~K}$. Предполагая, что гармоническое и ангармоническое приближения дают близкие результаты при комнатных температурах, $B\left(T_{0}\right) \approx B_{\mathrm{h}}\left(T_{0}\right)$, мы определяем значения $B(T)$ по приведенным в [21] значениям $Y(T)$, а затем вычисляем $Ф Д В \mathrm{e}^{-2 W(\kappa)}$ в ангармоническом приближении, используя формулу (35).

ТАБлицА 3. ФДВ для железа, вычисленный при температуре $T=1373 \mathrm{~K}$ по формуле (33), т. е. в гармоническом приближении с дебаевской плотностью фоннонных состояний (столбец 2), и по формуле (35) с реальной плотностью состояний в гармоническом (столбец 3 ) и ангармоническом (столбец 4) приближениях.

\begin{tabular}{c|ccc}
\hline$\kappa, \AA^{-1}$ & \multicolumn{3}{|c}{$\mathrm{e}^{-2 W(\kappa)}$} \\
\hline 1.7 & 0.942 & 0.960 & 0.934 \\
3.0 & 0.833 & 0.881 & 0.809 \\
\hline
\end{tabular}

В табл. 3 приведены значения ФДВ для ОЦК-железа в гармоническом и ангармоническом приближениях при температуре $T=1373 \mathrm{~K}$. Как видно из таблицы, различия результатов гармонического приближения с дебаевской и реальной плотностями фононных состояний невелики (ср. столбцы 2 и 3). Различия между гармоническим и ангармоническим приближениями с реальной плотностью состояний в пределах зоны Бриллюэна также незначительны: при $\kappa=1.7 \AA^{-1}$ ФДВ дает поправку $4 \%$ в гармоническом и около $7 \%$ в ангармоническом приближениях (см. столбцы 3 и 4). При бо́льших значениях вектора рассеяния ангармонический вклад возрастает: так, при $\kappa=3 \AA^{-1}$ в гармоническом приближении поправка за счет ФДВ составляет $12 \%$, а в ангармоническом - примерно $19 \%$.

\section{4. ЗАКЛЮЧЕНИЕ}

Выведена формула, связывающая сечение рассеяния нейтронов с коррелятором потенциала, который описывает произвольное взаимодействие нейтрона с кристаллом. Предложен краткий и простой вывод выражения для ФДВ в гармоническом 
приближении в случае произвольного типа рассеяния. ФДВ для ферромагнитных металлов вычислен как функция модуля вектора рассеяния $\kappa$ и температуры $T$. Показано, что при значениях $\kappa$, лежащих в пределах зоны Бриллюэна, ФДВ дает несущественную поправку и температурная зависимость магнитных характеристик определяется главным образом тепловыми спиновыми флуктуациями. Однако при значениях $\kappa$, отвечающих эксперименту по поляризованному магнитному рассеянию для ферромагнитных металлов в парамагнитной области, поправка за счет ФДВ может достигать 20-30\%. При высоких температурах на примере железа показано, что ангармонический вклад в ФДВ может быть заметным, однако он требует громоздких вычислений. Результаты в значительной мере зависят от используемых приближений. Гармоническое приближение дает удовлетворительные значения ФДВ даже за пределами зоны Бриллюэна.

Полученные результаты могут быть использованы для оценки ФДВ в различных экспериментах по рассеянию нейтронов, а также в экспериментах по рентгеновской и мессбауэровской спектроскопии.

Благодарности. Авторы признательны рецензенту за полезные замечания.

\section{Список литературы}

[1] Т. Мория, Спиновые флуктуации в магнетиках с коллективизированными электронами, Мир, М., 1988.

[2] J. Schweizer, "Polarized neutrons and polarization analysis", Neutron Scattering from Magnetic Materials, ed. T. Chatterji, Elsevier, Amsterdam, 2006, 153-213.

[3] N. Plakida, High-Temperature Cuprate Superconductors: Experiment, Theory, and Applications, Springer Series in Solid-State Sciences, 166, Springer, Berlin, 2010.

[4] Г. В. Парадеженко, Н. Б. Мельников, Б. И. Резер, "Коррелятор спиновой плотности и его фурье-образ в динамической теории спиновых флуктуаций", Выч. мет. программирование, 17:4 (2016), 474-486.

[5] P. J. Brown, H. Capellmann, J. Déportes, D. Givord, K. R. A. Ziebeck, "Observations of ferromagnetic correlations at high temperatures in paramagnetic iron", J. Magn. Magn. Mater., 30:2 (1982), 243-248.

[6] J. Hubbard, "Panel discussion on itinerant electron magnetism", Physics of Transition Metals (University of Leeds, 18-22 August, 1980), Institute of Physics Conference Series, 55, ed. P. Rhodes, IOP, Bristol, 1981, 669-687.

[7] H. A. Mook, "Neutron Scattering Studies of Magnetic Excitations in Itinerant Magnets", Spin Waves and Magnetic Excitations, eds. A. S. Borovik-Romanov, S. K. Sinha, Elsevier, Amsterdam, 1988, 425-478.

[8] N. B. Melnikov, B. I. Reser, V.I. Grebennikov, "Extended dynamic spin-fluctuation theory of metallic magnetism", J. Phys.: Condens. Matter, 23:27 (2011), 276003, 11 pp.

[9] N. B. Melnikov, B. I. Reser, "Short-range order above the Curie temperature in the dynamic spin-fluctuation theory", J. Magn. Magn. Mater., 397 (2016), 347-351.

[10] N. B. Melnikov, B. I. Reser, G. V. Paradezhenko, "Spin-density correlations in the dynamic spin-fluctuation theory: Comparison with polarized neutron scattering experiments", J. Magn. Magn. Mater., 411 (2016), 133-139.

[11] Н. Б. Мельников, Г. В. Парадеженко, Б. И. Резер, "Корреляции спиновой плотности и магнитное нейтронное рассеяние в ферромагнитных металлах", ТMФ, 191:1 (2017), $151-171$.

[12] L. Van Hove, "Time-dependent correlations between spins and neutron scattering in ferromagnetic crystals", Phys. Rev., 95:6 (1954), 1374-1384.

[13] Ю. А. Изюмов, Р. П. Озеров, Магнитная нейтронография, Наука, М., 1966.

[14] G. L. Squires, Introduction to the Theory of Thermal Neutron Scattering, Dover, New York, 1996. 
[15] N.D. Mermin, "A short simple evaluation of expressions of the Debye-Waller form", J. Math. Phys., 7:6 (1966), 1038.

[16] C.G. Windsor, "Neutron scattering experiments on itinerant electron magnets", Physica $B+C, 91$ (1977), 119-129.

[17] Ч. Киттель, Введение в физику твердого тела, Наука, М., 1978.

[18] P. J. Brown, H. Capellmann, J. Déportes, D. Givord, K. R. A. Ziebeck, "Ferromagnetic correlations in both the $\alpha$ and $\gamma$-phases of paramagnetic iron", J. Magn. Magn. Mater., 30:3 (1983), 335-339.

[19] P. J. Brown, H. Capellmann, J. Déportes, D. Givord, S. M. Johnson, K. R. A. Ziebeck, "The paramagnetic response of nickel at high temperature", J. Phys. France, 47:3 (1986), 491-496.

[20] A. A. Maradudin, P.A. Flinn, "Anharmonic contributions to the Debye-Waller factor", Phys. Rev., 129:6 (1963), 2529-2547.

[21] L.P. Pathak, J. Prakash, V.P. Singh, M.P. Hemkar, "Debye-Waller factors of $\alpha$-iron and sodium. II Willis's anharmonic theory", Aust. J. Phys., 28:6 (1975), 707-713.

[22] H. L. Kharoo, O. P. Gupta, M. P. Hemkar, "Temperature dependence of Debye-Waller factors in bcc metals. I", J. Phys. Soc. Japan, 43:6 (1977), 2030-2035.

[23] B. T. Willis, "Lattice vibrations and the accurate determination of structure factors for the elastic scattering of X-rays and neutrons", Acta Cryst. A, 25:2 (1969), 277-300.

[24] F. D. Vila, J. J. Rehr, H.H. Rossner, H. J. Krappe, "Theoretical x-ray absorption Debye-Waller factors", Phys. Rev. B, 76:1 (2007), 014301, 11 pp.

[25] G. V. Paradezhenko, N. B. Melnikov, B. I. Reser, Lattice vibrations in the harmonic approximation, arXiv: 1711.04302.

[26] А. Марадудин, Э. Монтролл, Дж. Вейсс, Динамическая теория кристаллической решетки в гармоническом приближении, Мир, М., 1965.

[27] R. J. Elliott, "Theory of neutron scattering by conduction electrons in a metal and on the collective-electron model of a ferromagnet", Proc. Roy. Soc. London Ser. A, 235:1202 (1956), 289-304.

[28] K. R. A. Ziebeck, P. J. Brown, "Measurement of the paramagnetic response function in the weak itinerant magnetic compound MnSi using polarised neutron scattering", J. Phys. F: Met. Phys., 10:9 (1980), 2015-2024.

[29] Дж. Займан, Принципь теории твердого тела, Мир, М., 1974.

[30] J. J. Quinn, K.-S. Yi, Solid State Physics: Principles and Modern Applications, Springer, Berlin, 2009.

[31] K.-H. Hellwege, J. L. Olsen (eds.), Metals: Phonon States. Electron States and Fermi Surfaces. Phonon States of Elements. Electron States and Fermi Surfaces of Alloys, Landolt-Börnstein - Group III Condensed Matter, 13A, Springer, Berlin, 1981.

[32] J. Crangle, G. M. Goodman, "The magnetization of pure iron and nickel", Proc. Roy. Soc. London Ser. A, 321:1547 (1971), 477-491.

[33] W. B. Pearson, Handbook of Lattice Spacings and Structures of Metals and Alloys, Pergamon Press, New York, 1958.

[34] D. J. Kim, New Perspectives in Magnetism of Metals, Kluwer, New York, 1999.

[35] N. B. Melnikov, B. I. Reser, "Magnetism of metals in the dynamic spin-fluctuation theory", Phys. Metalls Metallogr., 117:13 (2016), 1328-1383.

[36] P. J. Brown, H. Capellmann, J. Déportes, D. Givord, S. M. Johnson, J. W. Lynn, K.R. A. Ziebeck, "The magnetic response of paramagnetic Fe at high energy transfers", J. Phys. France, 46:5 (1985), 827-830.

[37] A. V. Ruban, S. Khmelevskyi, P. Mohn, B. Johansson, "Temperature-induced longitudinal spin fluctuations in Fe and Ni", Phys. Rev. B, 75:5 (2007), 054402, 7 pp.

[38] P.-W. Ma, S. L. Dudarev, "Longitudinal magnetic fluctuations in Langevin spin dynamics", Phys. Rev. B, 86:5 (2012), 054416, 10 pp. 